\title{
Epidemiological surveys of, and research on, soil-transmitted helminths in Southeast Asia: a systematic review
}

\author{
Julia C. Dunn ${ }^{1,2^{*}}$, Hugo C. Turner ${ }^{1,2}$, Aung Tun ${ }^{3}$ and Roy M. Anderson ${ }^{1,2}$
}

\begin{abstract}
Soil-transmitted helminth (STH) infections of humans fall within the World Health Organization's (WHO) grouping termed the neglected tropical diseases (NTDs). It is estimated that they affect approximately 1.4 billion people worldwide. A significant proportion of these infections are in the population of Southeast Asia. This review analyses published data on STH prevalence and intensity in Southeast Asia over the time period of 1900 to the present to describe age related patterns in these epidemiological measures. This is with a focus on the four major parasite species affecting humans; namely Ascaris lumbricoides, Trichuris trichiura and the hookworms; Necator americanus and Ancylostoma duodenale. Data were also collected on the diagnostic methods used in the published surveys and how the studies were designed to facilitate comparative analyses of recorded patterns and changes therein over time. PubMed, Google Scholar, EMBASE, ISI Web of Science, Cochrane Database of Systematic Reviews and the Global Atlas of Helminth Infections search engines were used to identify studies on STH in Southeast Asia with the search based on the major key words, and variants on, "soil-transmitted helminth" "Ascaris" "Trichuris" "hookworm" and the country name. A total of 280 studies satisfied the inclusion criteria from 11 Southeast Asian countries; Brunei, Cambodia, Indonesia, Lao People's Democratic Republic (Lao PDR), Malaysia, Myanmar, Philippines, Singapore, Thailand, Timor-Leste and Vietnam. It was concluded that the epidemiological patterns of STH infection by age and species mix in Southeast Asia are similar to those reported in other parts of the world. In the published studies there were a large number of different diagnostic methods used with differing sensitivities and specificities, which makes comparison of the results both within and between countries difficult. There is a clear requirement to standardise the methods of both STH diagnosis in faecal material and how the intensity of infection is recorded and reported in future STH research and in monitoring and evaluation (M\&E) of the impact of continuing and expanding mass drug administration (MDA) programmes.
\end{abstract}

Keywords: Soil-transmitted helminths, Southeast Asia, Systematic review, Neglected tropical diseases, Mass drug administration, Monitoring and evaluation

\section{Background}

Soil-transmitted helminth (STH) infections belong to the so called neglected tropical diseases (NTDs) that affect human populations in poorer regions of the world [1]. Their presence is a typical marker of poverty where access to sanitation and clean water is limited and, concomitantly, standards of hygiene are low [2-5]. There

\footnotetext{
* Correspondence: julia.dunn@imperial.ac.uk

${ }^{1}$ London Centre for Neglected Tropical Disease Research, London, UK

${ }^{2}$ Department of Infectious Disease Epidemiology, School of Public Health,

Faculty of Medicine, Imperial College London, London, UK

Full list of author information is available at the end of the article
}

are four main species of STH; namely, Ascaris lumbricoides (roundworm), Trichuris trichiura (whipworm) and the hookworms (Ancylostoma duodenale and Necator americanus) [6].

It is estimated that over 1.4 billion people are infected with STHs $[2,7,8]$. According to a study by Pullan et al. [8], the highest number of STH infections occurs in Asia, where the People's Republic of China and India have the greatest concentration of people infected with intestinal worms. Southeast Asia is the region with the highest reported prevalences of STH infection in recent decades $[8,9]$. 
The countries of Southeast Asia have various attributes that contribute to the continually high prevalence of STH. For example, most Southeast Asian countries have a tropical and moist climate, which is ideal for the survival of STH eggs/larvae in the environment [10]. This environment acts to promote infection within the human population [10,11]. Socioeconomic factors such as lack of adequate water resources, sanitation and poor hygiene practices have repeatedly been proven to be related to high STH prevalence within a community [5] since transmission of A. lumbricoides and T. trichiura occurs via the faecal-oral route [6]. Several countries within Southeast Asia are amongst the poorest in the world, without adequate water and sanitation infrastructure $[10,12]$ and, therefore, the parasites prosper in such environments $[13,14]$.

The goal set by the World Health Organization (WHO) for STH control by 2020 is to reduce morbidity from STH in preschool-aged (pre-SAC: 2-5 years) and school-aged children (SAC: 5-14 years) to a level below which it would not be considered a public health problem [15]. Similarly, the target set by the 2012 London Declaration on NTDs, is to achieve preventive chemotherapy (PCT) coverage of $75 \%$ of all pre-SAC and SAC at risk of STH by $2020[16,17]$. To meet this goal, Southeast Asian countries that are endemic for STH have been conducting mass drug administration (MDA) campaigns $[13,18]$, treating pre-SAC and SAC in affected areas with antihelminthic drugs such as albendazole and mebendazole at regular intervals $[19,20]$. The current goals and objectives set by the WHO focus on reducing morbidity in pre-SAC and SAC, the age groups most commonly and most severely affected by two of the major STH infections; namely, A. lumbricoides and T. trichiura [15]. Hookworm is found at the highest intensities in adults, and hence, its abundance is not greatly affected by only treating pre-SAC and SAC [21-23]. At present, there is a growing interest in investigating the feasibility of interrupting the transmission of STH by broadening the range of ages targeted for treatment and increasing coverage in all age groups [24-27].

To evaluate the impact of MDA, comprehensive epidemiological studies need to be conducted periodically to measure changes in the prevalence and intensity of each STH species over time [28]. With the increasing focus on the effect that SAC-targeted MDA has on prevalence and intensity of STH across all ages [22, 29], ideally monitoring and evaluation (M\&E) of control impact should be based on epidemiological studies that are community wide. Also, to be able to compare progress between different regions and countries, the methods and design of M\&E should be standardised in terms of the diagnostic method used and the prevalence plus intensity measures made in the target population [30].
In this paper we review published epidemiological studies of STH in the Southeast Asia countries of Brunei, Cambodia, Indonesia, Lao People's Democratic Republic (Lao PDR), Malaysia, Myanmar, Philippines, Singapore, Thailand, Timor-Leste and Vietnam. The overall aim is to evaluate past STH publications from studies conducted in Southeast Asia and to help point to the ideal study design for the M\&E of control programme impact.

\section{Review}

This systematic review was developed in line with the Preferred Reporting Items for Systematic Reviews and Meta-Analyses (PRISMA) guidelines (see Checklist in Additional file 1).

\section{Selection criteria}

We include all published studies in English in which the prevalence and/or intensity of STH infection was measured in the Southeast Asia countries of Brunei, Cambodia, Indonesia, Lao PDR, Malaysia, Myanmar, Philippines, Singapore, Thailand, and Vietnam (the members of the Association of Southeast Asian Nations - ASEAN), within the period of January $1^{\text {st }}, 1900$ to July 2015. Timor-Leste was also included due to its proximity to the other Southeast Asian countries and since it is often included in public health analyses concerning Southeast Asia $[8,13]$. No studies were found concerning STH in Brunei. Observational and intervention studies were eligible for inclusion. We excluded studies that had the following criteria: (i) studies that did not report prevalence values for each STH separately (that just recorded prevalence of any STH); (ii) studies where the participants were selected from hospital in-patients; (iii) studies where the participants were not permanent residents of the specific country (e.g. refugees or migrants); (iv) duplicate publications or extension of analysis from an original study; and (v) studies where the full publication could not be obtained.

\section{Search strategy and methodology}

We identified published studies using automated database searches of EMBASE (1947 to July 2015), ISI Web of Science (1900 to July 2015), the National Library of Medicine's PubMed (1900 to July 2015) and further manual searching was done using Google Scholar, the Cochrane Database of Systematic Reviews (CDSR) and the website of the Global Atlas of Helminth Infections (GAHI - http://www.thiswormyworld.org/). We employed the following terms and variations on these terms: STH, or soil-transmitted helminth, or Ascaris, or Trichuris, or hookworm, or Ancylostoma, or Necator, or deworm. A full list of the search terms is provided in Additional file 1. We also searched the Global Neglected Tropical Diseases 
database [31] for data collected on STH from studies conducted in Southeast Asia but did not find any results.

Identified studies were exported into EndNote X6 (Thomson Reuters, New York, USA) for management. The abstracts of the studies were reviewed against the inclusion and exclusion criteria. The literature selection process is outlined in Fig. 1. Ultimately, 280 studies were identified that met the inclusion criteria, a full list of the included studies is provided in Additional file 2.

\section{Data extraction}

Data extraction included country name, study area, year the study was published, year the study was conducted, type of study (cross-sectional or longitudinal/cohort), sample size, age of participants, prevalence of each STH, intensity of each $\mathrm{STH}$, and diagnostic methods employed. If the publication did not state which year the study took place, the year of publication was used instead. Also, if the study was conducted over a range of years then the latest year of the stated range was used. For plotting the age distributions, the mid-point of the stated age range was used.

\section{Results and discussion}

\section{All identified studies}

A total of 280 studies were identified that met the inclusion criteria (Fig. 1). The breakdown of the number of studies by country is provided in Table 1 . Figure 2 illustrates the geographical distribution of study areas covered in the selected publications which met the inclusion criteria.

The largest proportion of the identified studies were conducted in Thailand (55 studies, $20 \%$ ), followed by Malaysia (51 studies, $18 \%$ ) and Indonesia (48 studies, $17 \%)$. Timor-Leste and Singapore had the fewest studies (one and two studies respectively). Two studies included

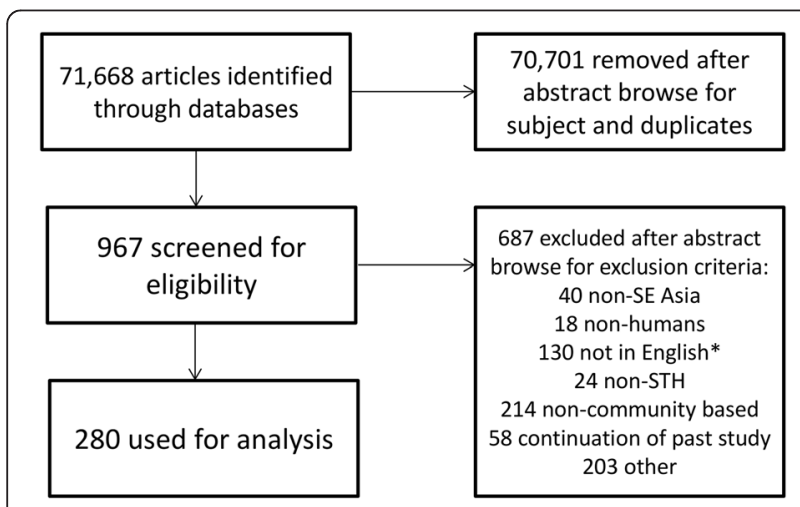

Fig. 1 Decision tree outlining the inclusion and exclusion criteria of the identified studies. *These papers could not be properly screened due to being published in non-English language journals and likely include studies in non-included countries such as Japan and South Korea data from two countries [32, 33] and one study included data from three countries [34]. Most of the studies identified were of a cross-sectional design (266 studies, $95 \%$ ) and the remaining studies were longitudinal (14 studies, $5 \%$ ). Figure 3 illustrates the distribution of the identified studies across time - the first study being undertaken in 1947. The number of published studies has increased steadily since 1947 to the present. In 2003 there was a marked increase in STH studies published perhaps due to an increased focus on the NTDs globally as a result of WHO guidance [18]. Another recent spike in the number of STH studies published coincides with the London Declaration on NTDs in 2012 [17]. Both of these events signalled an increase in funding and materials to combat $\mathrm{STH}$, including for example the donation of albendazole tablets by GSK. The surge in published studies around these times, plus the longer term increasing trend, suggests a growing interest in STH control.

A large proportion of the selected studies measured STH prevalence and intensity from more than one study area within the same publication. Hookworm was, marginally, the most studied STH (256 studies), followed by A. lumbricoides (251 studies) and T. trichiura was the least studied (241 studies). This went against the expectation that hookworm would be the least studied of the STHs in Southeast Asia, as the climate of sub-Saharan Africa is more suitable for hookworm transmission, whereas the warm and humid climate of Southeast Asia is ideal for A. lumbricoides and T. trichiura $[9,10]$. Conversely, it seems that most identified studies were inclusive of all STH species.

\section{Diagnostic methods}

The differences in the method of STH diagnosis and quantification of intensity used across the selected studies made it difficult to compare studies. For example, STH prevalence was measured by 13 different methods (Fig. 4). Of the 280 studies, 40 reported using more than one method for diagnosing STH infection, whilst eight studies did not report the method used at all. The specificity and sensitivity of the different methods of STH diagnosis have been analysed in a number of publications and have been found to vary widely [30, 35-37]. Therefore, it can be inferred that the accuracy of the prevalence and intensity results in STH studies also varies over time. However, it is difficult to quantify this due to the lack of standardised procedures.

The Kato-Katz technique was the most frequently used method of STH diagnosis (128 studies (45.7\%), including studies that used multiple methods). The proportion of studies using each type of diagnostic method has changed over time, with the proportion of studies using Kato-Katz and the formalin-ether concentration (FEC) method increasing since the 1980s. The current 
Table 1 Breakdown of the identified studies

\begin{tabular}{|c|c|c|c|c|c|}
\hline Country & $\begin{array}{l}\text { Number } \\
\text { of studies }\end{array}$ & $\begin{array}{l}\text { Number of prevalence studies } \\
\text { that measured intensity }\end{array}$ & $\begin{array}{l}\text { Number of studies with } \\
\text { prevalence by age groups }\end{array}$ & $\begin{array}{l}\text { Number of studies with } \\
\text { age groups and intensity }\end{array}$ & $\begin{array}{l}\text { Number of studies with full } \\
\text { age distribution and intensity }\end{array}$ \\
\hline Cambodia & 22 & 1 & 4 & 0 & 0 \\
\hline Indonesia & 48 & 9 & 30 & 6 & 4 \\
\hline Lao PDR & 26 & 2 & 10 & 1 & 1 \\
\hline Malaysia & 51 & 11 & 20 & 6 & 1 \\
\hline Myanmar & 22 & 4 & 9 & 4 & 4 \\
\hline Philippines & 37 & 7 & 13 & 1 & 1 \\
\hline Singapore & 2 & 0 & 1 & 0 & 0 \\
\hline Thailand & 55 & 4 & 13 & 4 & 4 \\
\hline $\begin{array}{l}\text { Timor- } \\
\text { Leste }\end{array}$ & 1 & 0 & 0 & 0 & 0 \\
\hline Vietnam & 20 & 5 & 5 & 2 & 2 \\
\hline TOTAL & $280^{a}$ & 43 & 105 & 24 & 17 \\
\hline
\end{tabular}

${ }^{a}$ Includes two studies that had components in two different countries, and one study that had components in three different countries

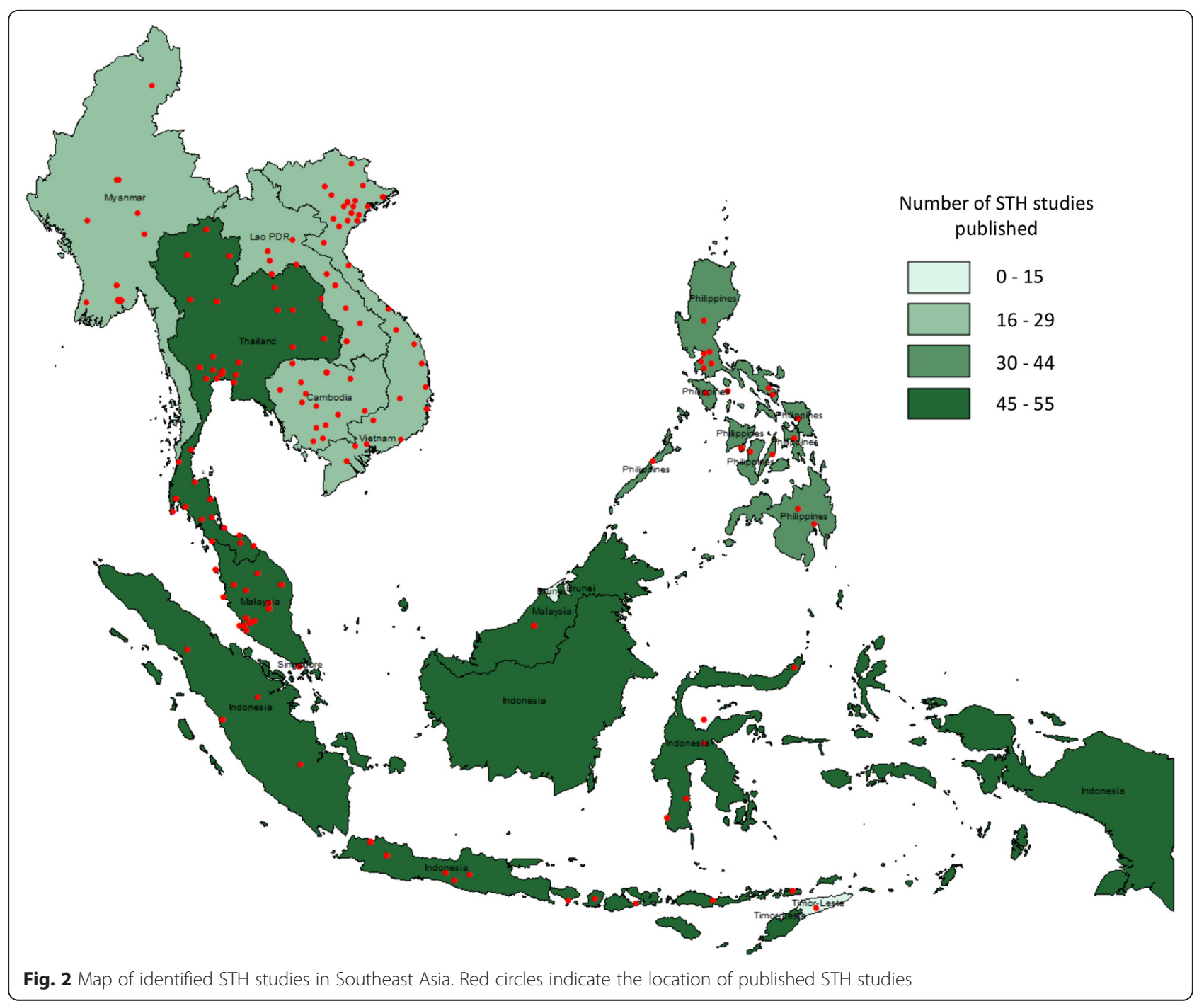




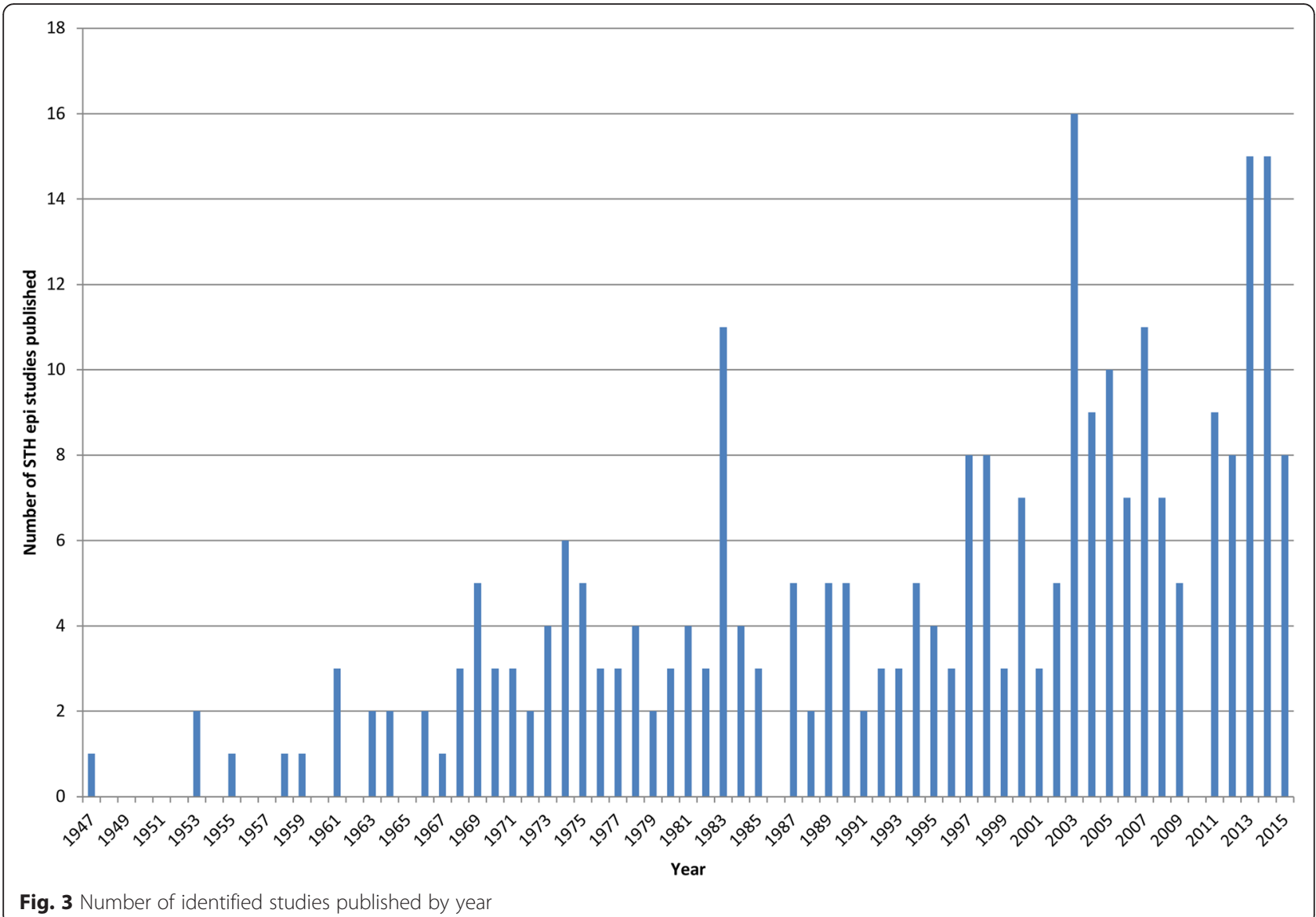

decade has also seen the first studies using more advanced molecular and immunological diagnostic tools such as the polymerase chain reaction (PCR) [38-40] and enzyme-linked immunosorbent assay (ELISA) [41]. In each decade there was a small proportion of studies that do not specify which method the authors used to diagnose STH infection.

There was also variation in how the diagnostic methods were applied. For example, variation between studies is apparent where the Kato-Katz method was used as the primary diagnostic method. Studies varied in how many Kato-Katz thick smears were prepared from each participant, how many times these slides were read (for quality control) [42] and whether or not readings were by the same operator or different people. Out of the 128 studies that used Kato-Katz, 97 (75.8 \%) did not clearly state that any repetition had been made, 11 $(8.6 \%)$ studies stated that they prepared/read Kato-Katz slides once, 19 (14.8\%) studies in duplicate and one study in the Philippines [43] read Kato-Katz slides six times.

\section{Prevalence and intensity metrics}

Most control programmes use prevalence as their main epidemiological indicator, as advised by the WHO $[15,19]$.
However, prevalence as an indicator $[21,44,45]$ is far from ideal given the highly non-linear relationship between this measure and the average intensity of infection when parasite distributions of worms or eggs per gram (EPG) output per host is aggregated (negative binomial) in form [46].

Figure 5 shows overall STH prevalence plotted against average intensity for the studies that measured intensity by EPG counts. There are clearly discernible relationships between STH prevalence and average intensity displayed in these plots. Prevalence is non-linearly related to average intensity where the former changes rapidly at low intensities, but slowly at high intensities. The importance of this relationship lies in the observation that large changes in intensity, possibly caused by the effects of MDA, are not well measured by changes in prevalence. Therefore, M\&E for MDA programmes must be based on intensity. The precise relationship between the two epidemiological measures is determined by the magnitude of the negative binomial aggregation parameter $k$ (which varies inversely with the degree of aggregation). For high aggregation prevalence plateaus well below $100 \%$, while for low degrees of aggregation of worms it quickly saturates to high prevalence figures [46]. The 


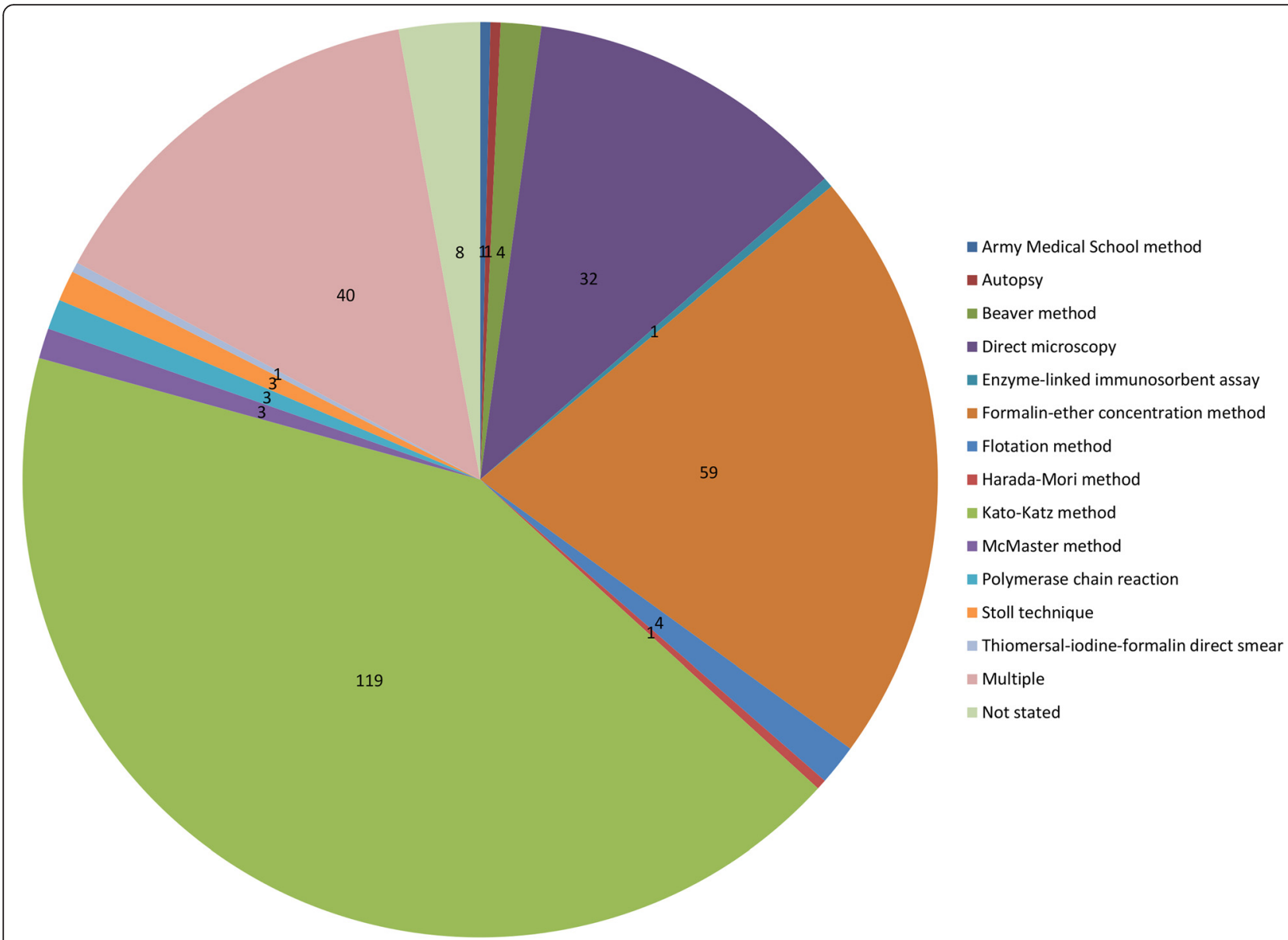

Fig. 4 Number of studies that used each STH diagnostic method. Multiple = studies that reported using multiple methods of diagnosis

heterogeneity displayed in this non-linear relationship may be due in part to the inclusion of data from different settings, countries and decades on the same graph. The vertical lines on Fig. 5 indicate the boundaries of the intensity groupings defined by the WHO as low, medium and high [15]. Note that for values within one intensity classification (low, medium or high) the prevalence of infection varies widely. This reflects differing degrees of worm aggregation within the various human communities studied. For example, the prevalence values for hookworm in the low intensity group (low intensity group mean EPG 414.42) ranged from close to 0 to $94 \%$.

As specified in the inclusion criteria, all of the studies selected reported at least one prevalence value for a species of STH. Of these 280 studies, only 43 (15\%) also measured STH intensity of infection in the study participants. Table 1 details the number of studies that measured intensity of STH in participants. Similar to prevalence, STH intensity was measured using a variety of different methods between studies. In 29 studies intensity was measured using the indirect method of mean EPG of faeces. Eight additional studies used EPG but presented the geometrical means only. Five studies used the more direct method of mean worm burden based on worm expulsion in faeces post chemotherapy. A single study used eggs per millilitre (EPM) of faeces as the measure of intensity.

\section{Age distributions of prevalence and intensity of infection}

The current focus of STH control, determined by the goals set by the WHO [15], is reducing morbidity in pre-SAC and SAC, the age groups that suffer the highest morbidity from heavy A. lumbricoides and T. trichiura infection $[18,47]$. Consequently, most studies are focussed on STH infection in these age groups. However, recent mathematical model-based studies of STH transmission dynamics [23, 29, 44, 48, 49] have helped focus attention onto the burden of infection in adults and how it affects overall STH transmission in a given community. These studies concluded that in many settings, transmission could not be interrupted by only SAC-focussed MDA, this is especially true for hookworm where prevalence and intensity is highest in the adult age groups [21,50]. Therefore, in many instances morbidity control will not lead to elimination, as adults will not be treated and transmission 


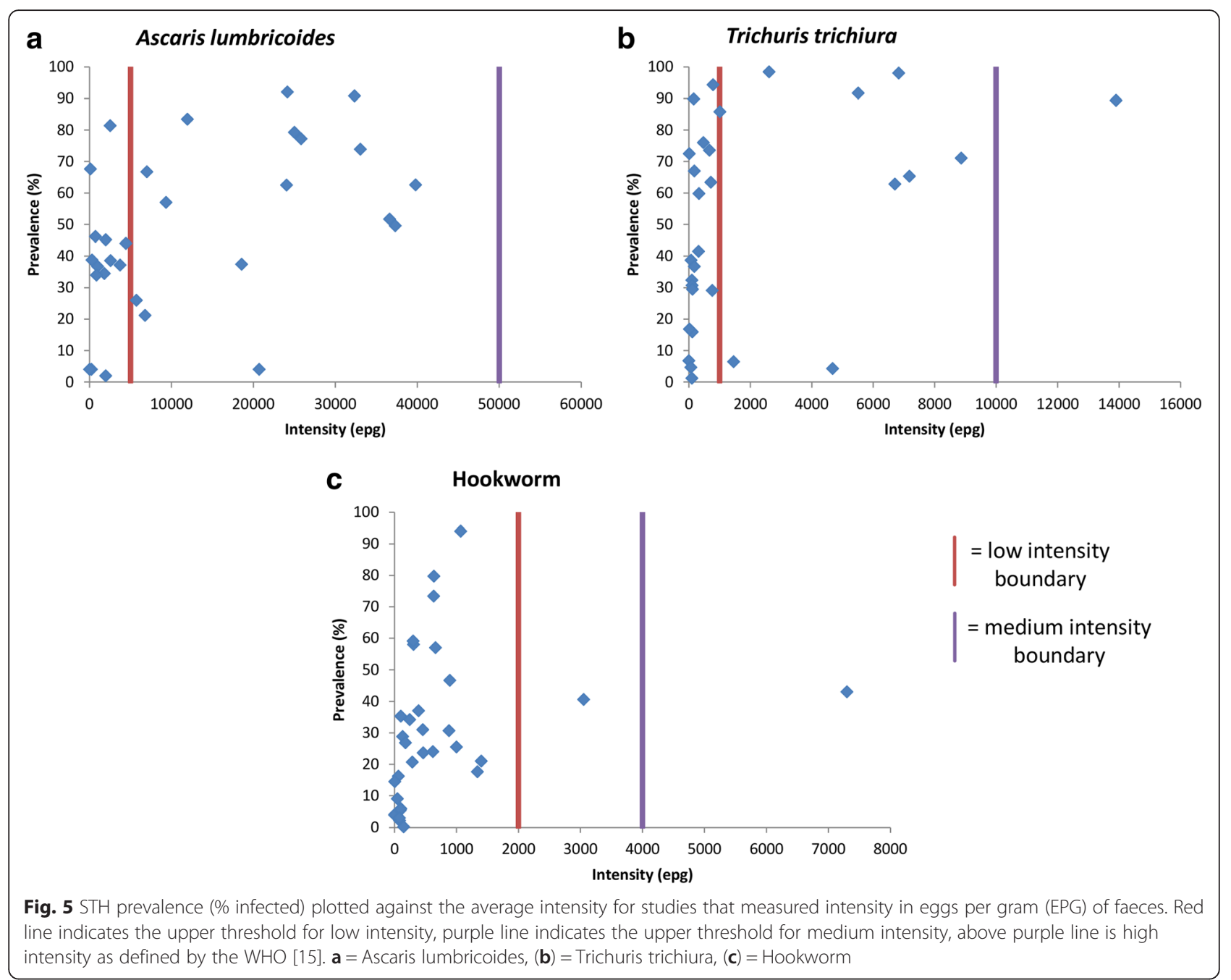

will not be broken. To break transmission, country MDA programmes may have to adapt to include all age groups. As such, there is a need for comprehensive epidemiological studies measuring prevalence and intensity across all age groups.

Table 2 presents the details of the 17 studies that measured STH prevalence and intensity of infection in all age groups within a community ( $6 \%$ of the total number of identified studies). The small number of these studies is indicative of the lack of comprehensive epidemiological M\&E of control impact of MDA on STH in the Southeast Asia region. Cambodia, Singapore and TimorLeste did not have any studies that fit these criteria. Even within these 17 studies, there is substantial variation in how prevalence and intensity are measured and summarised (Table 2).

Figure 6 shows the prevalence age distribution of each STH for two example countries, the Philippines [51] and Thailand [52-55]. Prevalence of A. lumbricoides and T. trichiura is higher for the included Philippines study
(Fig. 6a and b). A. lumbricoides prevalence peaks in the SAC groups in both the Philippines and Thailand studies. Hookworm prevalence for both Philippines and Thailand increases to a peak around the 20-29 age groups (Fig. $6 \mathrm{c}$ and $\mathrm{f}$ ), and remains high across the older age groups. However, T. trichiura prevalence varies between the two countries; prevalence in the Philippines study is lower for pre-SAC (Fig. 6b) but remains at high level for the other age groups, whereas in the Thailand study (Fig. 6e) prevalence is similarly lower in pre-SAC but then peaks in SAC and decreases over the older age groups. Additional file 1: Figure S1 presents these data for each country included in the review.

Figure 7 records the age distributions of the mean intensity of infection for the studies that recorded faecal EPG, the most commonly used measurement excepting a few studies that employed worm expulsion methods. The plots contain data from studies in all Southeast Asia countries included in the analysis (except for Singapore and Timor-Leste which did not have any studies that 
Table 2 Details of the studies that measured intensity across all ages

\begin{tabular}{|c|c|c|c|c|c|c|}
\hline First Author & Year & Country & Sample size & Parasite species & Diagnostic method & Intensity measure \\
\hline Bakta & 1993 & Indonesia & 2331 & hk & KK & EPG \\
\hline Higgins & 1984 & Indonesia & 227 & asc, tri, hk & MM & EPG \\
\hline Joe & 1959 & Indonesia & 664 & asc, tri, hk & AUT & Mean worm burden \\
\hline Margono & 1983 & Indonesia & 276 & asc, tri, hk & KK & EPG \\
\hline Phongluxa & 2013 & Lao PDR & 574 & asc, tri, hk & KK & EPG (geo mean) \\
\hline Rahman & 1994 & Malaysia & 204 & asc, tri, hk & KK & EPG \\
\hline Yogore & 1953 & Philippines & 229 & asc, tri, hk & DM & EPM \\
\hline Preuksaraj & 1983 & Thailand & 43341 & asc, tri, hk & KK/ST & EPG \\
\hline Sadun & 1953 & Thailand & 219 & hk & $\mathrm{DM} / \mathrm{ST}$ & EPM \\
\hline Sadun & 1955 & Thailand & 13469 & hk & $\mathrm{DM} / \mathrm{ST}$ & EPG \\
\hline Bethony & 1998 & Thailand & 641 & hk & NS & EPG \\
\hline King & 2005 & Vietnam & 201 & asc & ELISA & EPG \\
\hline Needham & 1998 & Vietnam & 543 & asc, tri, hk & KK & EPG \\
\hline Hlaing & 1990 & Myanmar & 2826 & asc & $\mathrm{DM} / \mathrm{FEC} / \mathrm{KK}$ & EPG \\
\hline Hlaing & 1984 & Myanmar & 783 & asc & $\mathrm{DM} / \mathrm{FEC} / \mathrm{KK}$ & EPG \\
\hline Hpay & 1970 & Myanmar & 571 & asc, tri & $\mathrm{DM} / \mathrm{FEC} / \mathrm{ST}$ & Mean worm burden \\
\hline Tu & 1970 & Myanmar & 671 & asc, tri & $\mathrm{DM} / \mathrm{FEC} / \mathrm{ST}$ & Mean worm burden \\
\hline
\end{tabular}

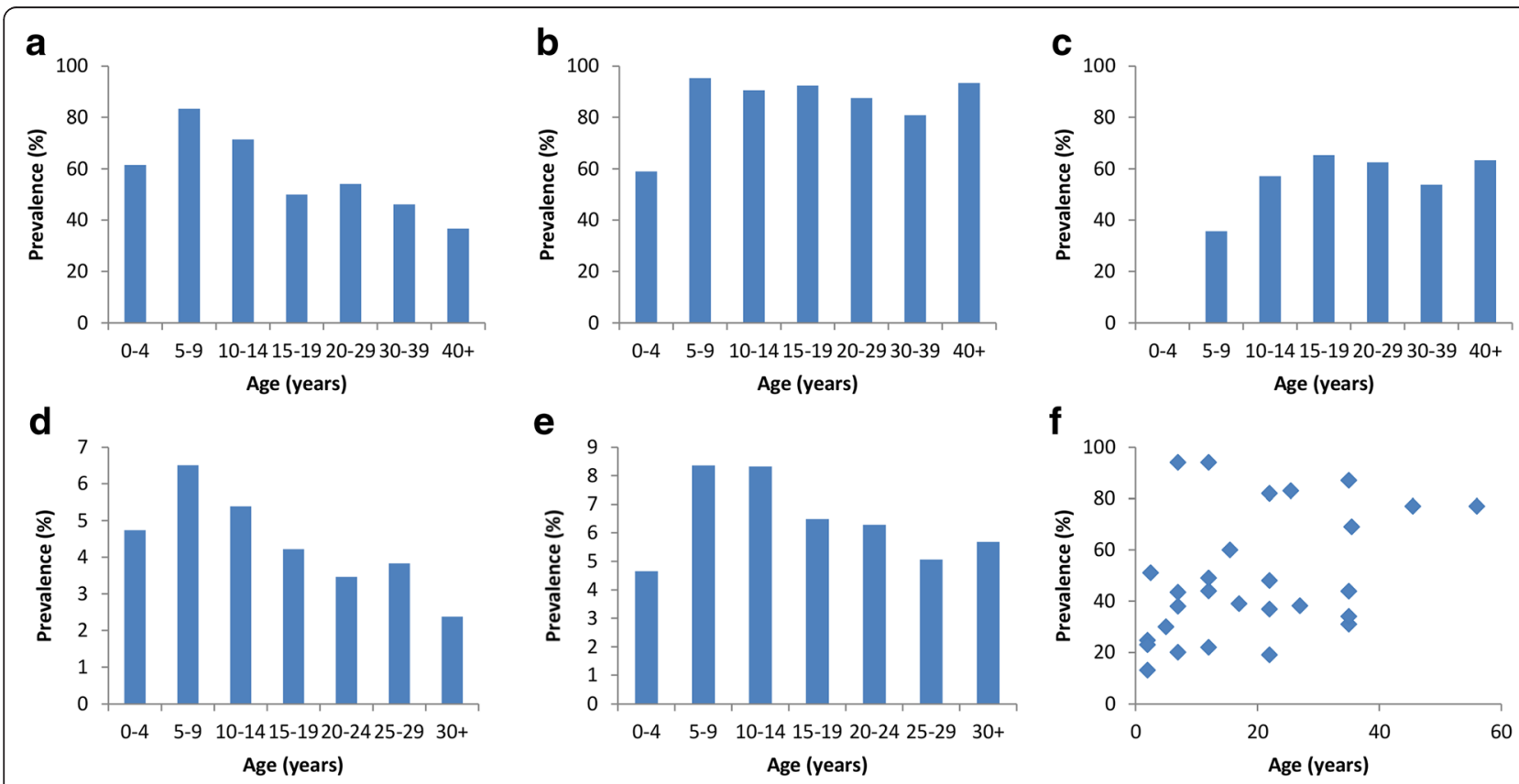

Fig. $\mathbf{6}$ Age distribution of the prevalence of infection for studies in the Philippines ( $\mathbf{a}, \mathbf{b}$ and $\mathbf{c}$ ) and Thailand ( $\mathbf{d}$, e and $\mathbf{f}$ ) that measured intensity and prevalence across all age groups. Figure $(\mathbf{f})$ includes data from more than one study and data points were plotted against the mid-point of the reported age group. $A=$ Philippines, Ascaris lumbricoides. $B=$ Philippines, Trichuris trichiura. $C=$ Philippines, hookworm. $D=$ Thailand, Ascaris lumbricoides. $\mathrm{E}=$ Thailand, Trichuris trichiura. $\mathrm{F}=$ Thailand, hookworm. Note different scales on figure (e) and (f) 


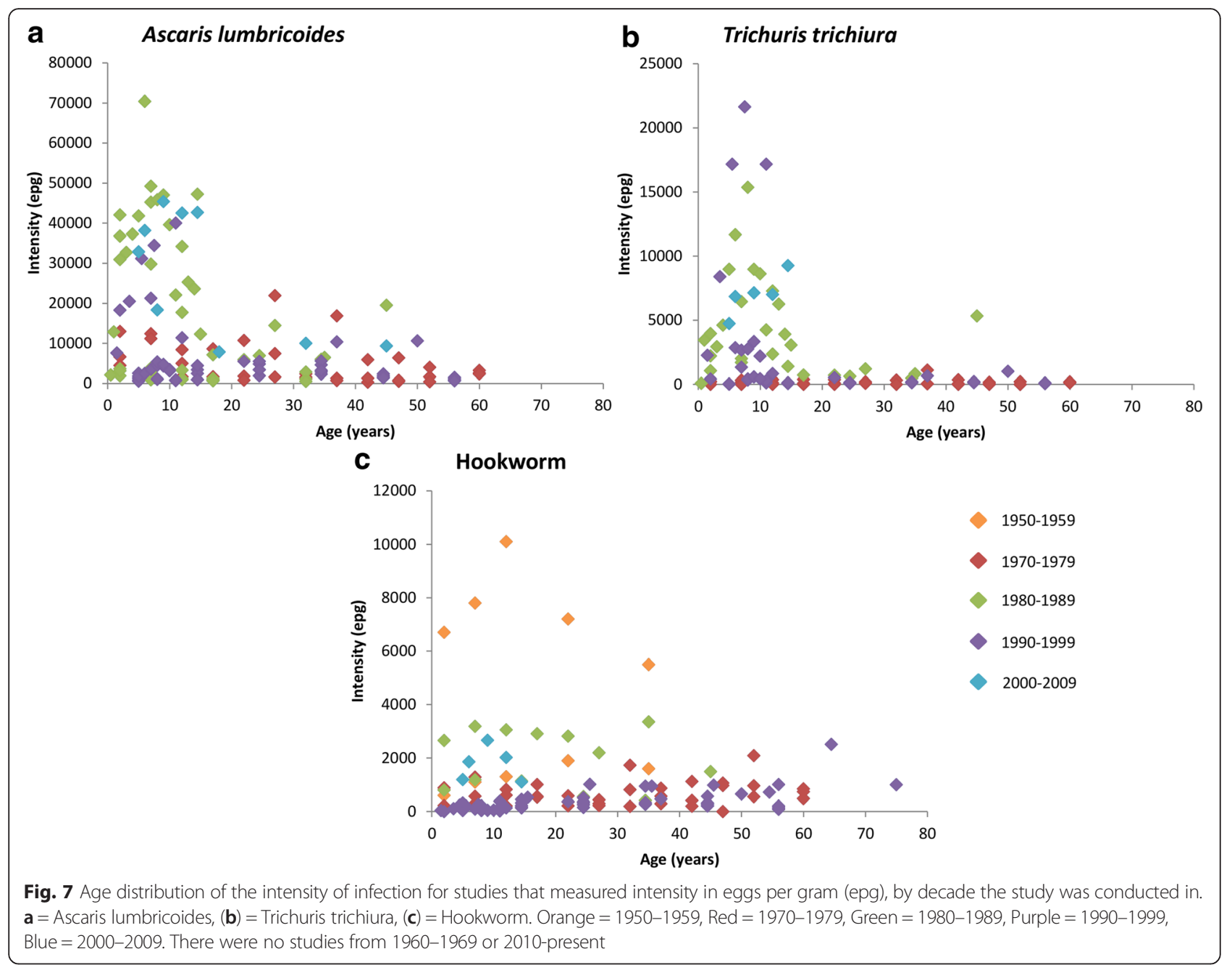

included intensity values). A. lumbricoides and T. trichiura show convex curves with higher mean intensities in the younger age groups (pre-SAC and SAC), which then decreased with increasing age. Hookworm intensities increased with age and then plateaued in older age groups. However, in a few studies there are several data points that denote high intensities in pre-SAC and SAC age groupings. Past comprehensive epidemiological studies have indicated that there is usually a higher intensity of hookworm in adult age groups [21, 50]. A possible reason for this pattern not being seen in this analysis is due to the under-representation of adults in the people sampled in the included studies. For example four studies included hookworm intensity values for SAC [56-59] and none for adults. Also, the age groupings are usually much larger for the adults (e.g. all ages above 40 years or all ages above 50 years) and so may not represent the variation between different adult age groups on a finer age range and the possible increase in hookworm burden in the later years of life. It is relevant to note that very few studies have been conducted post-2000 that record prevalence and intensity of infection across a broad range of age classes - and yet this period to the present is when MDA coverage has been rising. M\&E programmes in Southeast Asia, to record the impact of MDA, need to focus on intensity across all age groupings.

Intensity of infection can also be measured by a more direct method, mean worm burden, based on worm expulsion post-chemotherapy (Fig. 8). As this method directly counts the number of worms harboured by a participant, there is perhaps little uncertainty of the person's intensity level, assuming that stool collection is complete and lasts for many days post-treatment. However, there are far fewer studies that use mean worm burden as the intensity measure as well as reporting these intensities by age and including a representation of the entire community [60-63]. All but one of these studies are in Myanmar. The remaining study [60] was carried out in Indonesia and was the only case in this review to collect data via autopsy. The data for T. trichiura was not included in Fig. 8 because, in two studies, the adult age groups were grouped in a manner that does not adequately reflect the variation 

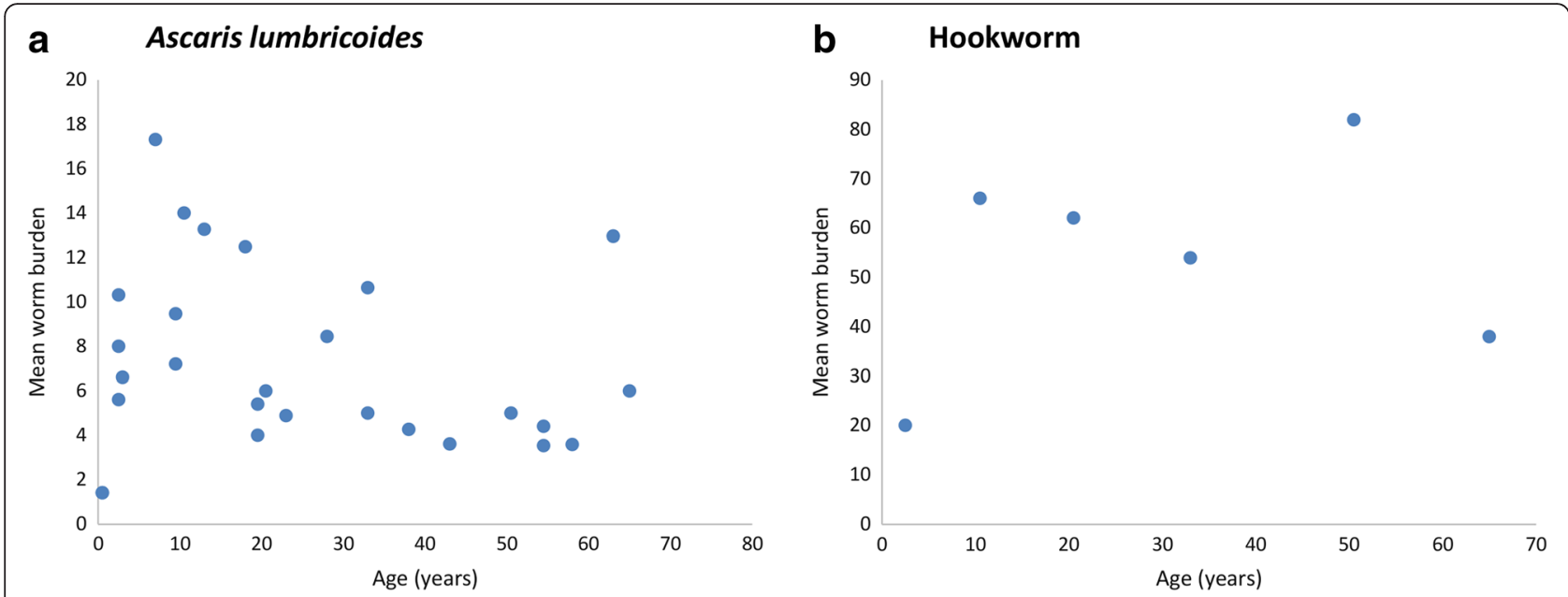

Fig. 8 Age distribution of intensity for studies that measured intensity in mean worm burden. $\mathbf{a}=$ Ascaris lumbricoides, $(\mathbf{b})=$ Hookworm

across adult groups (all ages above 14 years). For A. lumbricoides mean worm burden has a small peak around SAC, decreases around $30-50$ years old and then increases again at the oldest ages (Fig. 8a). Therefore, in terms of worm intensity, the older age groups also suffer from a high worm burden, although the morbidity in adults may be less damaging than in SAC. However for hookworm, for which there is only one study [60], mean worm burden peaks in the $30-40$ years individuals (Fig. 8b).

\section{Limitations}

The first limitation of our review, which has been highlighted earlier in this paper, is our inability to directly compare studies due to the differences in diagnostic techniques and units of measurement. However, despite this lack of standardisation, when the studies were pooled and analysed, trends in prevalence and intensity across the age classes could be determined. The age distributions of $A$. lumbricoides, T. trichiura and hookworm prevalence (Fig. 6) displayed a similar pattern to those recorded in past studies and reviews $[46,50,61,64]$. However, in future research and in M\&E appraisals it is obviously highly desirable to place greater emphasis on the standardisation of the methods of measurement.

Secondly, the significance of changes in the prevalence and intensity of STH infection over time can best be interpreted when examined in conjunction with knowledge of the history of control efforts, specifically MDA, in the area under consideration. It is possible to determine this history at country-level via the WHO PCT databank [20], but the data only extends as far back as 2003 and is incomplete for a number of countries. Regional-level MDA data and information on other interventions, such as water and sanitation hygiene (WASH) and behaviour change, is required before the cause of any changes in prevalence and/or intensity can be established. Figure 9 plots the average (over the reviewed Southeast Asian countries) change in MDA coverage as recorded in the WHO PCT database in pre-SAC and SAC. The raw data, as extracted from the WHO PCT databank interactive report, is presented in Additional file 3. Note that average coverage increases from 2003 to 2013 in both age groupings as a trend in the region. However, considerable differences exist between countries as recorded in the raw data. It seems likely that this overall rise in coverage is the main driver behind the trend for a decrease in prevalence and intensity of STH infection in most countries in the region.

Finally, whilst every effort has been made to make this review comprehensive and inclusive of all the studies published on STH in Southeast Asia, it is inevitable that some studies will have been missed due to them not being published in English language journals. In the future, it would be useful to have these papers translated to add further information to the review. It is likely however, that the inclusion of these papers would have supported the conclusions drawn in this paper. In general, STH studies are difficult to compare and contrast due to a lack of standardisation in diagnostic methods.

\section{Conclusions}

Since 1947 there have been a large number of epidemiological studies on STH in Southeast Asia, increasing in numbers over the years to the present day. However, only a small proportion of these studies can be truly deemed comprehensive in the sense of good coverage of prevalence and intensity data over a broad range of age classes in the studied communities or areas. Only 17 studies (6 \%) measured both STH prevalence and intensity across all age groups. It is imperative for the future of STH control in Southeast Asia that more studies are completed with much better, standardised M\&E design. Ideal M\&E design should include a consistent diagnostic 


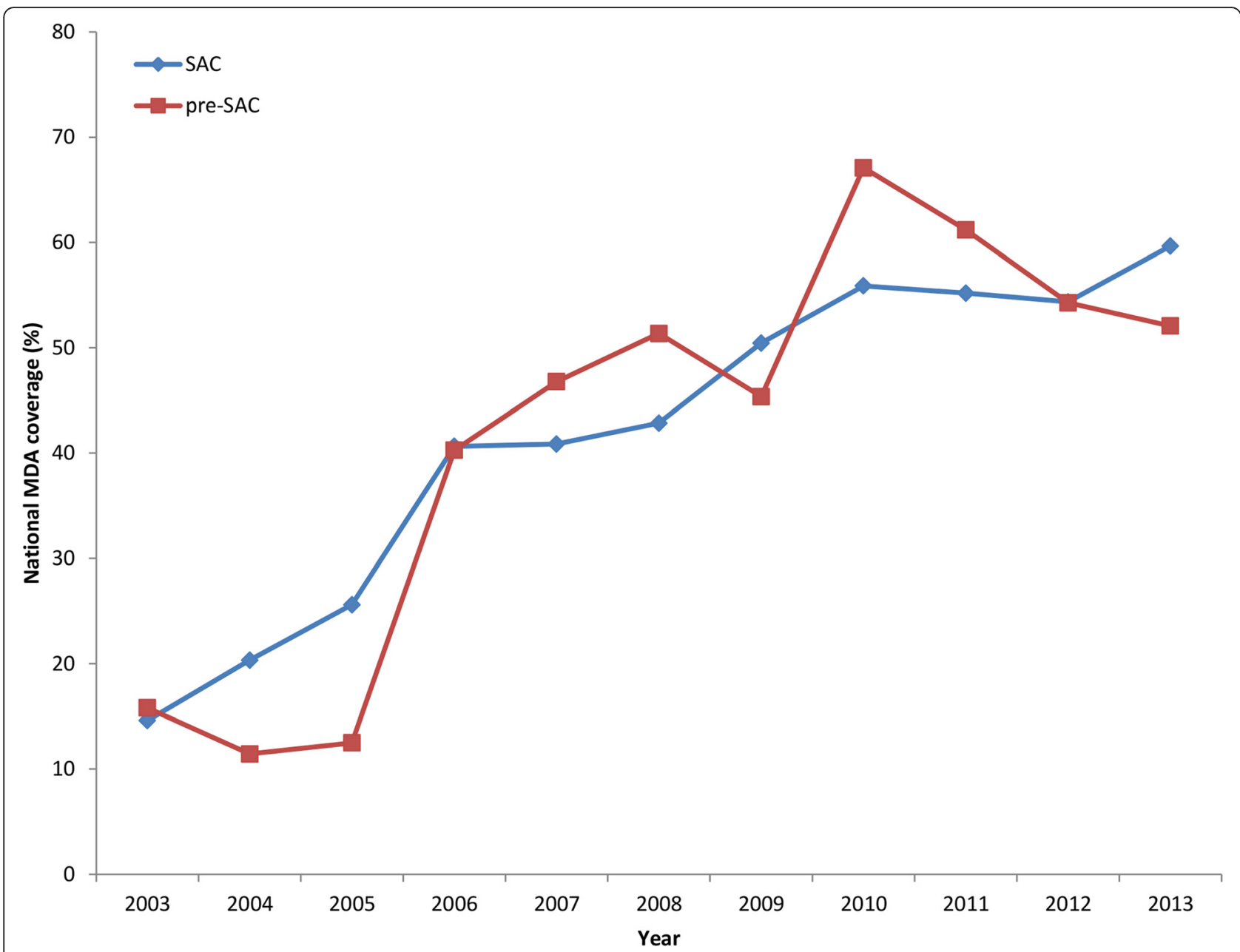

Fig. 9 Mean mass drug administration (MDA) coverage for pre-school aged children (pre-SAC) and school-aged children (SAC) in the Southeast Asian countries. Red = pre-SAC, Blue = SAC. Data collated from the World Health Organization preventive chemotherapy databank [20]. Country-specific data is included in Additional file 3

method. Currently Kato-Katz is recommended by the WHO due to its ease of use in the field and low cost [19]. However, methods with higher sensitivity and specificity are desirable as the impact of expanded MDA programmes drive infection to low levels. M\&E should use STH intensity as the primary measure to determine the effectiveness of MDA programmes, including those integrated with WASH, education and nutrition components. Large changes in intensity, reflecting effective treatment, are not well measured by changes in prevalence. M\&E should also cover the different ecological areas of a country and should be repeated at set intervals. They should be continued for a specified time after MDA, and any other control activities, have been stopped. The data is of obvious importance in evaluating the progress and effectiveness of MDA programmes, plus the impact of treating pre-SAC and SAC on transmission within the total community. Finally, this data can also be of great value in mathematical modelling studies to determine the most effective targeting by age group and frequency of MDA to interrupt transmission of STH in different populations and settings.

Perhaps the most important message arising from this review is that public health workers must place greater emphasis on the standardisation of diagnostic methods for determining STH prevalence and intensity. The achievement of some degree of uniformity in epidemiological methods would mean that studies can be directly compared both within and between countries to effectively evaluate and compare the progress of control efforts. To achieve this, guidance and support from organisations such as WHO to endemic countries would be useful for emphasising the importance of comprehensive and continuous M\&E. Indeed it would be desirable if WHO played the lead role in setting standardisation guidelines. Training in best practice and uniform methods could help lower income countries optimise M\&E. It has proven difficult in the past for researchers to agree on one method of 
STH diagnosis. There have been numerous recent papers evaluating the sensitivity and specificity of the various methods $[30,65,66]$. However, the new technologies of quantitative PCR, multiplex PCR and ELISA offer great potential especially in low prevalence areas, as MDA impact increases, to identify the remaining pockets of transmission.

\section{Additional files}

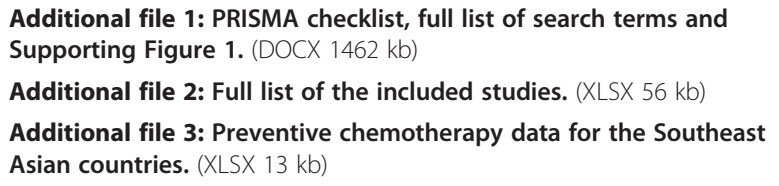

\section{Abbreviations}

Asc: Ascaris lumbricoides; ASEAN: Association of Southeast Asian Nations; AUT: autopsy method; CDSR: Cochrane Database of Systematic Reviews; DM: Direct Microscopy method; ELISA: enzyme-linked immunosorbent assay; EPG: eggs per gram of faeces; EPG (geo mean): eggs per gram of faeces (geometric mean); EPM: eggs per millilitre of faeces; FEC: formalin-ether concentration; GAHI: Global Atlas of Helminth Infections; Hk: hookworm; KK: Kato-Katz method; Lao PDR: Lao People's Democratic Republic; M\&E: monitoring and evaluation; MDA: mass drug administration; MM: McMaster method; NS: not stated; NTD: neglected tropical disease; PCR: Polymerase Chain Reaction; PCT: preventive chemotherapy; PreSAC: Preschool-aged children; PRISMA: Preferred Reporting Items for Systematic Reviews and Meta-analyses; SAC: school-aged children; SE Asia: Southeast Asia; ST: stoll technique; STH: soil-transmitted helminth; Tri: Trichuris trichiura; WASH: water and sanitation hygiene; WHO: World Health Organization.

\section{Competing interests}

Roy M Anderson is a non-executive Director of GlaxoSmithKline (GSK). GlaxoSmithKline played no role in study design, data collection and analysis, decision to publish, or preparation of the manuscript.

\section{Authors' contributions}

$J C D$ conducted the literature search, data collection and analysis. JCD and HCT drafted the first version of the manuscript. JCD, HCT and RMA contributed to the design of the study. JCD, HCT, AT and RMA contributed to the writing of the paper. All authors read and approved the final version of the manuscript.

\section{Acknowledgements}

Roy Anderson gratefully acknowledges funding of the NTD Modelling Consortium by the Bill and Melinda Gates Foundation in partnership with the Task Force for Global Health. The views, opinions, assumptions or any other information set out in this article are solely those of the authors.

\section{Funding}

JCD and HCT are supported by the London Centre for Neglected Tropical Disease Research (funded by GSK). RMA is supported by the Bill \& Melinda Gates Foundation (\#OPP1053230).The funders had no role in study design, data collection and analysis, decision to publish, or preparation of the manuscript.

\section{Author details}

'London Centre for Neglected Tropical Disease Research, London, UK. ${ }^{2}$ Department of Infectious Disease Epidemiology, School of Public Health, Faculty of Medicine, Imperial College London, London, UK. ${ }^{3}$ Worm Free Myanmar Kids Program, Tun Khit Foundation, Yangon, Myanmar.

Received: 12 November 2015 Accepted: 8 January 2016 Published online: 27 January 2016

\section{References}

1. Pullan RL, Brooker SJ. The global limits and population at risk of soil-transmitted helminth infections in 2010. Parasit Vectors. 2012;5(1):81.

2. Hotez PJ, Fenwick A, Savioli L, Molyneux DH. Rescuing the bottom billion through control of neglected tropical diseases. Lancet. 2009;373(9674):1570-5.

3. Esrey SA, Potash JB, Roberts L, Shiff C. Effects of improved water supply and sanitation on ascariasis, diarrhoea, dracunculiasis, hookworm infection, schistosomiasis, and trachoma. Bull World Health Organ. 1991;69(5):609-21.

4. Ziegelbauer K, Speich B, Mäusezahl D, Bos R, Keiser J, Utzinger J. Effect of sanitation on soil-transmitted helminth infection: systematic review and meta-analysis. PLoS Med. 2012;9(1):e1001162.

5. Strunz EC, Addiss DG, Stocks ME, Ogden S, Utzinger J, Freeman MC. Water, sanitation, hygiene and soil-transmitted helminth infection: a systematic review and meta-analysis. PLoS Med. 2014;11(3):e1001620.

6. Bethony J, Brooker S, Albonico M, Geiger SM, Loukas A, Diemert D, et al. Soil-transmitted helminth infections: ascariasis, trichuriasis, and hookworm. Lancet. 2006;367(9521):1521-32.

7. Hotez PJ, Molyneux DH, Fenwick A, Kumaresan J, Ehrlich-Sachs S, Sachs JD, et al. Control of neglected tropical diseases. New Engl J Med. 2007; 357:1018-27.

8. Pullan RL, Smith JL, Jasrasaria R, Brooker SJ. Global numbers of infection and disease burden of soil transmitted helminth infections in 2010. Parasit Vectors. 2014;7(1):37.

9. Utzinger J, Bergquist R, Olveda R, Zhou XN. Important helminth infections in Southeast Asia. Diversity, potential for control and prospects for elimination. Adv Parasitol. 2010;72(C):1-30.

10. Brooker S, Singhasivanon P, Waikagul J, Supavej S, Kojima S, Takeuchi T, et al. Mapping soil-transmitted helminths in Southeast Asia and implications for parasite control. Southeast Asian J Trop Med. 2003;34(1):24-36.

11. Matsusaki G. Influence of low temperature, sunshine, desiccation and excreta upon the eggs of Ancylostoma Duodenale and Necator Americanus. ii. Sunshine. Yokohama Med Bull. 1963;14:123-6.

12. WHO. Report of the informal consultation on scaling up treatment of soil transmitted helminthiasis in the South-East Asia region: 10-11 October 2011, New Delhi, India. New Delhi: World Health Organization; 2012.

13. Jex AR, Lim YAL, Bethony JM, Hotez PJ, Young ND, Gasser RB. Soil-transmitted helminths of humans in Southeast Asia-towards integrated control. Adv Parasitol. 2011:74(C):231-65

14. Hotez PJ, Ehrenberg JP. Escalating the global fight against neglected tropical diseases through interventions in the Asia Pacific Region. Adv Parasitol. 2010;72(C):31-53.

15. WHO. Eliminating soil-transmitted helminthiases as a public health problem in children: progress report 2001-2010 and strategic plan 2011-2020. Geneva: World Health Organization; 2012.

16. WHO. Neglected tropical diseases - prevention, control, elimination and eradication. WHA 66.20. In: Sixty-Sixth World Health Assembly. Geneva: World Health Organization; 2013.

17. The London Declaration [http://unitingtocombatntds.org/resource/londondeclaration]. Accessed 14 May 2015.

18. WHO. In: Crompton D, Montresor A, Nesheim M, Savioli L, editors. Controlling disease due to helminth infections. Geneva: WHO; 2003.

19. WHO. Helminth control in school-age children - Second Edition. Geneva: World Health Organization; 2012.

20. WHO. PCT databank: soil-transmitted helminthiases. Geneva: World Health Organization; 2014.

21. Anderson RM, Schad GA. Hookworm burdens and faecal egg counts: an analysis of the biological basis of variation. Trans R Soc Trop Med Hyg. 1985;79(6):812-25.

22. Anderson RM, Truscott JE, Easton A, Oliveira R, Brooker S, Hollingsworth D. How frequently and to whom should mass chemotherapy be administered to control soil transmitted helminths? In: American Society for Tropical Medicine and Hygiene Annual Meeting Presentations, November 2013. Washington DC, USA; 2013. 151.

23. Turner H, Truscott J, Bettis A, Shuford K, Dunn J, Hollingsworth T, et al. An economic evaluation of expanding hookworm control strategies to target the whole community. Parasit Vectors. 2015;8(1):570.

24. Boatin BA, Basañez MG, Prichard RK, Awadzi K, Barakat RM, Garcia HH, et al. A research agenda for helminth diseases of humans: towards control and elimination. PLoS Negl Trop Dis. 2012;6(4):e1547.

25. Brooker SJ, Nikolay B, Balabanova D, Pullan RL. Global feasibility assessment of interrupting the transmission of soil-transmitted helminths: a statistical modelling study. Lancet Infect Dis. 2015;15(8):941-50. 
26. Global Partners are taking the "Neglect" out of "Neglected Tropical Diseases" [http://www.gatesfoundation.org/Media-Center/Press-Releases/2014/04/ Global-Partners-Are-Taking-the-Neglect-out-of-Neglected-Tropical-Diseases]

27. Lo NC, Bogoch II, Blackburn BG, Raso G, N'Goran EK, Coulibaly JT, et al. Comparison of community-wide, integrated mass drug administration strategies for schistosomiasis and soil-transmitted helminthiasis: a cost-effectiveness modelling study. Lancet Glob Health. 2015;3(10):e629-38.

28. Ohta N, Waikagul J. Disease burden and epidemiology of soil-transmitted helminthiases and schistosomiasis in Asia: the Japanese perspective. Trends Parasitol. 2007;23(1):30-5.

29. Truscott JE, Hollingsworth TD, Brooker SJ, Anderson RM. Can chemotherapy alone eliminate the transmission of soil transmitted helminths. Parasit Vectors. 2014;7(1):266

30. Levecke B, Behnke JM, Ajjampur SSR, Albonico M, Ame SM, Charlier J, et al. A comparison of the sensitivity and fecal egg counts of the McMaster egg counting and Kato-Katz thick smear methods for soil-transmitted helminths. PLoS Negl Trop Dis. 2011;5(6):e1201.

31. Hürlimann E, Schur N, Boutsika K, Stensgaard A-S, Laserna de Himpsl M, Ziegelbauer K, et al. Toward an open-access global database for mapping, control, and surveillance of neglected tropical diseases. PLoS Negl Trop Dis. 2011;5(12), e1404

32. Jatanasen S. A brief survey of parasitic helminth in south Laos and Cambodia with a comparison to the state in Thailand. Jpn J Med Sci Biol. 1961;14(5-6):257-62

33. Cross JH, Basaca-Sevilla V. Intestinal parasitic infections in Southeast Asia. Southeast Asian J Trop Med. 1981;12(2):262-74.

34. Kabayashi J, Socheat D, Phommasack B, Tun A, Huy Nga N. The Asian Center of International Parasite Control (ACIPAC): Five years of achievement IV: Activities in partner countries (Cambodia, Lao PDR, Myanmar and Vietnam): small scale pilot project (SSPP) and other impacts. Southeast Asian J Trop Med. 2005;36(S3):28-40.

35. Sayasone S, Utzinger J, Akkhavong K, Odermatt P. Repeated stool sampling and use of multiple techniques enhance the sensitivity of helminth diagnosis: a cross-sectional survey in southern Lao People's Democratic Republic. Acta Trop. 2015;141(PB):315-21.

36. Tarafder MR, Carabin H, Joseph L, Balolong Jr E, Olveda R, McGarvey ST. Estimating the sensitivity and specificity of Kato-Katz stool examination technique for detection of hookworms, Ascaris lumbricoides and Trichuris trichiura infections in humans in the absence of a 'gold standard'. Int J Parasitol. 2010;40(4):399-404.

37. Nikolay B, Brooker SJ, Pullan RL. Sensitivity of diagnostic tests for human soil-transmitted helminth infections: a meta-analysis in the absence of a true gold standard. Int J Parasitol. 2014;44(11):765-74.

38. Schär F, Odermatt P, Khieu V, Panning M, Duong S, Muth $S$, et al. Evaluation of real-time PCR for Strongyloides stercoralis and hookworm as diagnostic tool in asymptomatic schoolchildren in Cambodia. Acta Trop. 2013;126(2):89-92.

39. Wiria AE, Hamid F, Wammes L, Kaisar MMM, May L, Prasetyani MA, et al. The Effect of Three-Monthly Albendazole Treatment on Malarial Parasitemia and Allergy: A Household-Based Cluster-Randomized, Double-Blind, Placebo-Controlled Trial. PLoS ONE. 2013;8(3):e57899.

40. Gordon CA, McManus DP, Acosta LP, Olveda RM, Williams GM, Ross AG, et al. Multiplex real-time PCR monitoring of intestinal helminths in humans reveals widespread polyparasitism in Northern Samar, the Philippines. Int $J$ Parasitol. 2015;45(7):477-83.

41. King E, Kim H, Dang N, Michael E, Drake L, Needham C, et al. Immunoepidemiology of Ascaris lumbricoides infection in a high transmission community: antibody responses and their impact on current and future infection intensity. Parasite Immunol. 2005;27(3):89-96.

42. Speich B, Ali SM, Ame SM, Albonico M, Utzinger J, Keiser J. Quality control in the diagnosis of Trichuris trichiura and Ascaris lumbricoides using the Kato-Katz technique: experience from three randomised controlled trials. Parasit Vectors. 2015;8(82):25652120.

43. Papier K, Williams GM, Luceres-Catubig R, Ahmed F, Olveda RM, McManus DP, et al. Childhood malnutrition and parasitic helminth interactions. Clin Infect Dis. 2014;59(2):234-43.

44. Anderson RM, Truscott JE, Pullan RL, Brooker SJ, Hollingsworth TD. How effective is school-based deworming for the community-wide control of soil-transmitted helminths? PLoS Negl Trop Dis. 2013;7(2), e2027.

45. Pullan RL, Kabatereine NB, Quinnell RJ, Brooker S. Spatial and genetic epidemiology of hookworm in a rural community in Uganda. PLoS Negl Trop Dis. 2010;4(6):e713.
46. Anderson RM, May RM. Helminth infections of humans: mathematical models, population dynamics, and control. Adv Parasitol. 1985;24.

47. Sayasone S, Utzinger J, Akkhavong K, Odermatt P. Multiparasitism and intensity of helminth infections in relation to symptoms and nutritional status among children: a cross-sectional study in southern Lao People's Democratic Republic. Acta Trop. 2015;141(PB):322-31.

48. Truscott J, Turner H, Anderson R. What impact will the achievement of the current World Health Organization targets for anthelmintic treatment coverage in children have on the intensity of soil transmitted helminth infections? Parasit Vectors. 2015;8:551.

49. Anderson RM, Turner HC, Truscott JE, Hollingsworth TD, Brooker SJ. Should the goal for the treatment of doil transmitted helminth (STH) infections be changed from morbidity control in children to community-wide transmission elimination? PLoS Negl Trop Dis. 2015;9(8), e0003897.

50. Bradley M, Chandiwana SK, Bundy DAP. The epidemiology and control of hookworm infection in the Burma Valley area of Zimbabwe. Trans R Soc Trop Med Hyg. 1993;87(2):145-7.

51. Yogore Jr M. Intestinal parasitism from health and welfare conditions at the Victorias Milling Company, Inc. Edited by Lara H. 1953.

52. Sadun EH. Studies on the distribution and epidemiology of hookworm, Ascaris, and Trichuris in Thailand. Am J Epidemiol. 1955;62(2):116-55.

53. Sadun EH, Vajrasthira S. Studies on intestinal parasitic infections in Cholburi Province (Thailand). Am J Trop Med Hyg. 1953;2(2):286-97.

54. Bethony JM. Epidemiology and immunology of Necator americanus infection in rural Thailand: the role of acquired immunity in determining infection intensity. PhD Thesis: State University of New York at Buffalo; 1998.

55. Preuksaraj S, Jeradit C, Sathitayathai A, Kijvannee S, Seedonrusmi T. Studies on prevalence and intensity of intestinal helminthic infections in the rural population of Thailand 1980-1981. Collected papers on the control of soil-transmitted helminthiases. 1983;2:54-8.

56. Pegelow K, Gross R, Pietrzik K, Lukito W, Richards AL, Fryauff DJ. Parasitological and nutritional situation of school children in the Sukaraja district, West Java, Indonesia. Southeast Asian J Trop Med. 1997;28(1):173-90.

57. Al-Mekhlafi MSH, Azlin M, Aini UN, Shaikh A, Sa'iah A, Fatmah MS, et al. Prevalence and distribution of soil-transmitted helminthiases among Orang Asli children living in peripheral Selangor, Malaysia. Southeast Asian J Trop Med. 2006;37(1):40-7.

58. Norhayati M, Zainudin B, Mohammod CG, Oothuman P, Azizi O, Fatmah MS. The prevalence of Trichuris, Ascaris and hookworm infection in Orang Asli children. Southeast Asian J Trop Med. 1997;28(1):161-8.

59. Rahman WA. Helminthic infections of urban and rural schoolchildren in Penang Island, Malaysia: implications for control. Southeast Asian J Trop Med. 1998;29(3):596-8.

60. Joe LK, Siang TK. Human intestinal helminths obtained from autopsies in Djakarta. Indonesia Am J Trop Med Hyg. 1959;8(4):122-5.

61. Hlaing T, Than S, Htay Htay A, Myint L, Thein Maung M. Epidemiology and transmission dynamics of Ascaris lumbricoides in Okpo village, rural Burma. Trans R Soc Trop Med Hyg. 1984;78(4):497-504.

62. Tu M, El-Zawahry MM, Kyi DO, Hlaing K, Hla-Gyaw S, Kyaw-Myint, et al. Epidemiological features of intestinal parasitoses in the inhabitants of Okpo village, Burma. Union Burma J Life Sci. 1970;3:247-67.

63. Hpay D, El-Zawahry MM, Maung L, Kyi DO, Dwe RS, Sein T, et al. Epidemiological features of intestinal parasitoses in Dayebo village, Burma. Union Burma J Life Sci. 1970;3:289-307.

64. Croll NA, Anderson RM, Gyorkos TW, Ghadirian E. The population biology and control of Ascaris lumbricoides in a rural community in Iran. Trans R Soc Trop Med Hyg. 1982;76(2):187-97.

65. Becker SL, Lohourignon LK, Speich B, Rinaldi L, Knopp S, N'Goran EK, et al. Comparison of the Flotac-400 dual technique and the formalin-ether concentration technique for diagnosis of human intestinal protozoon infection. J Clin Microbiol. 2011;49(6):2183-90.

66. Sinniah B. Daily egg production of Ascaris lumbricoides: the distribution of eggs in the faeces and the variability of egg counts. Parasitol. 1982;84(1):167-75. 